\title{
Corona Graphs for Astronomical Calcualtions using L-Cordial Labeling
}

\section{J. Arthy}

Abstract: In this work we examine L-Cordial Labeling for $. T \_n \Theta K_{-} 1, D T \_n \Theta K_{-} 1 \quad \llbracket, Q \rrbracket \_n \Theta K_{-}(1),, B \_(n, n) \Theta K_{-} 1$, C_m $\Theta P \_n$ Graphs Keywords: (LC).

Corona, Triangular Snake, Quadrilateral Snake, L-Cordial

AMS: Classification: $05 C 78$.

\section{INTRODUCTION}

For our study we use the graph $G=(p, q)$ which are finite, simple and undirected. Initially L- Cordial Labeling (LCL) was introduced in [7] and proved some graphs for the same labeling in [8,9] LCL.In [2,3] et al proved cube $\mathrm{Q}_{3}$, octahedron and special graph admits LCL. H- Related graph for square difference, Prime cordial and Cube difference labeling were studied in [1,6,14].In [15] Veena ShindeDeorde, studied H-Cordial and prime labeling .In $[10,11,12,13]$ Ponraj et.al proved snake graph and there corona admits difference cordial labeling. Detailed survey descriptions are given in [4]. Condition and results follow from [5] In this work we prove corona for some special graphs admits $L$-Cordial labeling.

\section{PRELIMINARIES}

1. If there is a bijection function $f: E(G) \rightarrow\{1,2, \ldots|E|\}$, thus the vertex label is induced as 0 if the biggest label on the incident edges is even and is induced as 1 , if it is odd and follows the condition that $\left|V_{f}(1)-V_{f}(1)\right| \leq 1$. Isolated vertices are not included for labeling here. A $L$-cordial graph is a graph which admits the above labeling.

2. The corona $A \odot B$ graph $G$ is formed by taking one copies of $A$ (which has $p$ points) $p$ copies of $B$ and then attaching the $i^{\text {th }}$ point of $A$ to every point in the $i^{\text {th }}$ copy of $B$.

\section{Theorem 1:}

$T_{n} O K_{1}$ admits L-Cordial Labeling.

\section{Proof:}

Consider $G=T_{n} \Theta K_{1}$ with

$V=\left\{u_{i}, x_{i}, y_{i}, w_{i} / i=1,2, \ldots, n\right\}$ and

$E=\left\{u_{i} x_{i} / 1 \leq i \leq n\right\} \cup\left\{u_{i} u_{i+1}, w_{i} u_{i+1}, w_{i} y_{i}, u_{i} w_{i} / 1 \leq i \leq n-1\right\}$ respectivedy.

Revised Manuscript Received on December 11, 2019

J. Arthy, Assistant Professor, Department of Science and Humanities, Bharath Institute of Higher Education and Research, India
The edge labeling is given as

$$
\begin{aligned}
& \text { For } i=1,2 \ldots n-1 \\
& g\left(w_{i} u_{i+1}\right)=4 n+i-3 \\
& g\left(u_{i} u_{i+1}\right)=2 n+i-1 \\
& g\left(w_{i} y_{i}\right)=n+i \\
& g\left(u_{i} w_{i}\right)=3 n+i-2 \\
& \text { For } i=1,2 \ldots n \\
& g\left(u_{i} x_{i}\right)=i
\end{aligned}
$$

Thus we have the vertex distribution as $V_{g}(0)=V_{g}(1)$ for all $\mathrm{n}$. Hence it's clear that $T_{n} \odot K_{1}$

admits L-Cordial Labeling. Illustration of $T_{4} \odot K_{1}$ is given in Figure below.

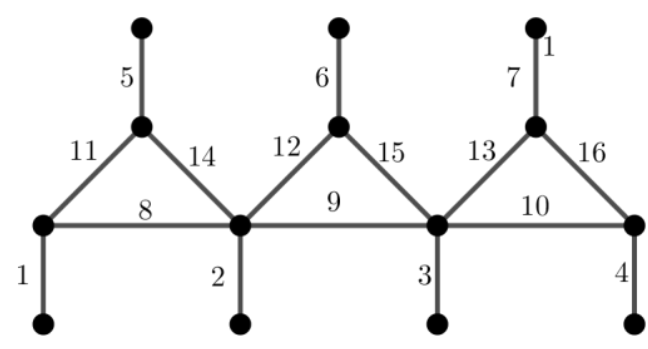

Theorem 2:

$D T_{n} \odot K_{1}$ is L-Cordial graph.

Proof:

Consider the graph $\mathrm{G}=D T_{n} \odot K_{1}$

With

$V\left(D T_{n} O K_{1}\right)=\left\{x_{j}, x_{j}^{s} 1 \leq j \leq n\right\} \cup\left\{w_{j}, w_{j}^{s}, y_{j}, y_{j}^{s} / 1 \leq j \leq\right.$ $n-1\}$

and

$E\left(D T_{n} \otimes K_{1}\right)=\left\{y_{j} y_{j}^{\prime}, w_{j} W_{j}^{\prime}, y_{j} x_{j+1}, w_{j} x_{j+1}, x_{j} W_{j}, x_{j} y_{j}, x_{j} x_{j+1} / 1 \leq j \leq n-1\right\} \cup\left\{x_{j} x_{j}^{\prime} / 1 \leq j \leq n\right\}$ We state the Labeling $f: E(G) \rightarrow\{1,2, \ldots ., 8 n-7\}$ as

When $n$ odd

For $j=1,2 \ldots, n-1$

$f\left(w_{j} w_{j}\right)=2 j$

$f\left(x_{j} x_{j+1}\right)=2 n-2+j \quad f\left(y y_{j}^{b}\right)=2 j-1$

$f\left(y_{j} x_{j+1}\right)=6 n-5+j$

$f\left(x_{j} y_{j}\right)=4 n+j-3$

$f\left(w_{j} x_{j+1}\right)=7 n-6+j$

$f\left(x_{j} w_{j}\right)=5 n+j-4$

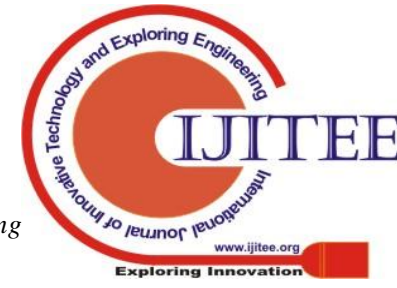


For $j=1,2 \ldots, n$

$f\left(x_{j} x_{j}\right)=3 n-3+j$

When $n$ is even

For $1 \leq j \leq n-1$

$f\left(w_{j} x_{j+1}\right)=3 n-3+j$

$f\left(x_{j} y_{j}\right)=j$

$f\left(x_{j} w_{j}\right)=n+j-1$

$f\left(x_{j} x_{j+1}\right)=4 n-4+j$

$f\left(y_{j} x_{j+1}\right)=2 n-2+j$

$f\left(w_{j} w_{j}\right)=5 n+2 j-5$

$f\left(y_{j} y_{j}\right)=5 n+2 j-6$

For $i=1,2 \ldots n$

$f\left(x_{j} x_{j}\right)=7 n-7+j$

Therefore from the above labeling it is clear that $V_{f}(0)=V_{f}(1)=3 n-2$ for all $\mathrm{n}$. Thus $D T_{n} \Theta K_{1}$ is

L-Cordial Graph. Illustration of $D T_{4} O K_{1}$ is given in Figure below.

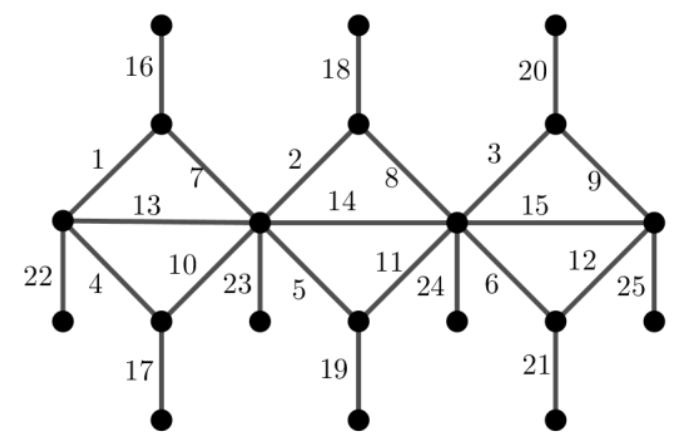

Theorem 3:

The Graph $Q_{n} \odot K_{1}$ is LC.

\section{Proof:}

Let $\mathrm{G}=Q_{\mathrm{n}} \odot K_{1}$ a graph with

$V=\left\{a_{j}, b_{j}, c_{j}, e_{j} / j=1,2 \ldots n-1\right\}$

$\cup\left\{a_{j}, d_{j} / j=1,2 \ldots n\right\}$ and

$$
E=\left\{\begin{array}{l}
a_{j} d_{j}, j=1,2 \ldots n \\
b_{j} e_{j}, a_{j} a_{j+1}, c_{j} e_{2 j}, a_{j} b_{j}, c_{j} a_{j+1}
\end{array}\right.
$$

For $j=1,2 \ldots n-f\left(x_{1} x_{n}\right)=m n$

Then we represent a one to one and onto

$f: E \rightarrow\{1,2, \ldots . q\}$ as

For $j=1,2 \ldots n$

$f\left(a_{j} d_{j}\right)=3 n-3+j$

For $j=1,2 \ldots . n-1$

$f\left(b_{j} e_{j}\right)=n+2 j-2$

$f\left(c_{j} e_{2 j}\right)=n+2 j-1$

$f\left(a_{j} a_{j+1}\right)=j$

$f\left(a_{j} b_{j}\right)=5 n-4+j$

$f\left(c_{j} a_{j+1}\right)=6 n-5+j$
Then it is easily observed that the above function satisfies the condition of L-Cordial Labeling.

Therefore $Q_{n} \odot K_{1}$ admits L-Cordial Labeling. Illustration of $Q_{5} \odot K_{1}$ is given in Figure below.

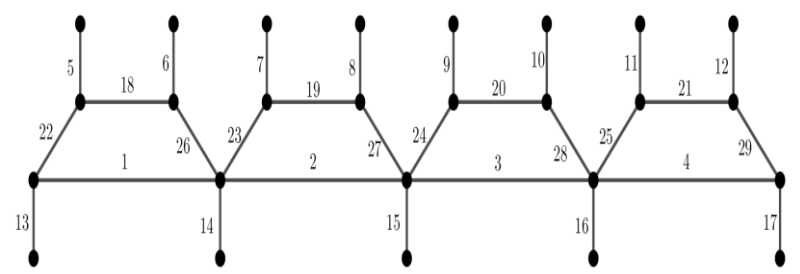

\section{Theorem 4:}

$B_{n n} \odot K_{1}$ admits LCL.

Proof:

Let $G=B_{n n} \odot K_{1}$ be a graph with

$V(G)=\left\{x_{v}, y, u_{\mathrm{i}}, v_{\mathrm{i}}, u_{\mathrm{i},}^{s}, v_{\mathrm{i}}^{s}, x^{s}, y^{s} / 1 \leq i \leq n\right\}$ and

$E(G)=\left\{x y, x x^{s}, y y^{s}, x u_{i}, y v_{i}, u_{i} u_{i,}^{s}, v_{i} v_{i}^{s} / 1 \leq i \leq n\right\}$

Then the edge labeling is given by

For $1 \leq i \leq n$

$f(x y)=1, f\left(x x^{5}\right)=2, f\left(y y^{5}\right)=3$

$f\left(x u_{i}\right)=2+2 i$

$f\left(y v_{i}\right)=3+2 i$

$f\left(u_{i} u_{i}^{1}\right)=2 n+2 i+2$

$f\left(v_{i} v_{i}^{1}\right)=2 n+2 i+3$

It is clear from the above defined labeling that $V_{f}(0)=V_{f}(1)$ for all $n$. Hence $B_{n, n} O K_{1}$ admits LCL.

\section{Theorem 5:}

Graph $C_{m} \odot P_{n}$ is L-Cordial graph.

Proof:

Let $G=C_{\mathrm{m}} \Theta P_{\mathrm{n}}$ with

$V(G)=\left\{x_{i}, y_{j}^{i} / 1 \leq i \leq m, 1 \leq j \leq n\right\}$

$E(G)=\left\{x_{i} y_{i+1} / 1 \leq i \leq m-1\right\} \cup\left\{x_{1} x_{n}\right\} \cup\left\{y_{j}^{i} y_{j+1}^{i} / 1 \leq i \leq m\right.$,

We define $f$ from $1,2, \ldots, q$ as

$f\left(x_{i} x_{i+1}\right)=m n-m+i, 1 \leq i \leq m-1$

$f\left(y_{j}^{i} y_{j+1}^{i}\right)=i+m(j-1), 1 \leq i \leq m, 1 \leq j \leq n-1$

Hence $C_{m} \odot P_{n}$ has $\left|V_{f}(0)-V_{f}(1)\right| \leq 1$ vertex. Thus $C_{\mathrm{m}} O P_{\mathrm{n}}$ is LCG.

\section{CONCLUSION}

In this paper we examined the determination of $L$-cordial behavior of $T_{n} \odot K_{1}, D T_{n} \odot K_{1}, Q_{n} \odot K_{1}$, $B_{n n} O K_{1}, C_{m} O P_{n}$. 


\section{REFERENCES}

1. J.Arthy, K.Manimekalai and K.Ramanathan, "Cube Difference Labeling Of Some Special Graphs”. AIP Conference Proceedings 2112, 020140 (2019).

2. J.Arthy, K.Manimekalai and K.Ramanathan , "Cycle And Path Related Graphs on L-Cordial Labeling". International journal of Innovative Science and Research Technology, Vol 4, issue 5, May 2019, ISSN No: 2456-2165.

3. J.Arthy, K.Manimekalai, K.Bhuvaneswari "On L-Cordial Graphs", Advancement in Engineering, Science and Technology. J.Mech.Cont.AMath.Sci, Special issue, N0-2, August (2019) PP 647-652.

4. Gallian. J.A,“A dynamic survey of graph labeling”, The Electronics journal of Combinatories, 17(2010) \#DS6.

5. Frank. Harary. "Graph theory" Narosa Publishing House (2001).

6. P.Jagadeeswari, K.Manimekalai and K.Ramanathan, "Square Difference Labeling Of $H$ -

7. Graphs" AIP Conference Proceedings 2112, 020147 (2019).

8. Mukund V.Bapat, "Some Vertex Prime Graphs and A New Type Of raph Labeling", International Journal Of Mathematics Trends and Technology (IJMTT), Vol 47, Number 1(2017),49-55.

9. Mukund, V.Bapat, "A Note On L-Cordial Labeling Of Graphs", International Journal Of Mathematics And Its Applications, Vol 5,Issue 4-D(2017),457-460.

10. Mukund V.Bapat, "New Results On L-Cordial Labeling Of Graphs", International Journal Of Statistics And Applied Mathematics,2018,3(2),641-644.

11. R. Ponraj, S. Sathish Narayanan, and R. Kala, Difference cordial labeling of corona graphs, J. Math. Comput. Sci., 3 (2013) 1237-1251.

12. R.Ponraj and S.Sathish narayanan "Difference Cordiality of some graphs obtained from Double Alternate Snake Graphs.”, Global Journal of Mathematical Sciences: Theory and Practical, Vol.5, No.3.(2013)PP.167-175.

13. R. Ponraj and S. Sathish Narayanan, Difference cordiality of some snake graphs, J. Appl. Math. and Informatics, 32 (2014) 377-387.

14. R. Ponraj and S. Sathish Narayanan, Difference cordial labeling of graphs obtained from triangular snakes, Applications Applied Math., 9(2) (2014) 811-825.

15. G.Subhashini, K.Manimekalai and K.Ramanathan, "Prime Cordial Labeling Of $H_{n} \odot K_{2}$

16. Graphs", AIP Conference Proceedings 2112, 020128 (2019).

17. Veena shinde-Deore, "Two Labeling Techniques for Corona Graph $C_{n} \Theta K_{1}$ ", International Journal Of Computer applications (0975-8887) Volume 64-No.12.Feb 2013.

\section{AUTHORS PROFILE}

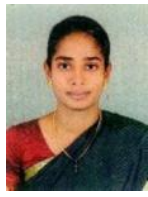

J. Arthy is an Assistant Professor, Department of Science and Humanities BIHER, Chennai. She is currently working on Graph labeling. She published 7 papers in international journal. 